\title{
LA REFORMA DE LA DIVISIÓN PROVINCIAL EN LA MESETA MERIDIONAL (1800-1850)
}

\author{
POR \\ JESÚS BURGUEÑO
}

A finales del siglo xvIII, la Meseta meridional se hallaba dividida en cinco provincias (figura 1) formadas a lo largo de la época moderna en torno a las ciudades con representación en Cortes, salvo La Mancha, que se instituyó en 1718 al establecerse el sistema de intendencias. Además del sinuoso trazado de los límites y de la pasmosa profusión de enclaves, era notoria la desproporción existente entre la gran extensión de Cuenca o La Mancha y la reducida superficie de Guadalajara y Madrid.

El proceso de homogeneización de esta caótica división provincial se inició bajo el reinado de Carlos IV. Fue entonces, veinte años antes de la primera división constitucional, cuando Madrid y Guadalajara adoptaron la forma básica que hoy presentan. Con todo, y al margen de las fluctuaciones de límites, la mayor innovación de la división castellano-manchega fue concebida en el Trieno liberal, cuando los comisionados del Gobierno, Felip Bauzá y José Agustín de Larramendi, dieron forma a la provincia de Albacete *.

Lógicamente, la reforma del mapa provincial de la Meseta meridional debe enmarcarse en el contexto general español, cuyos principales hitos, resumidos de manera sintética, fueron los siguientes:

\footnotetext{
Jesús Burgueño. Departamento de Geografía y Sociología. Universidad de Lleida

* El presente trabajo se inscribe en el proyecto de investigación PS95-0050 financiado por la Dirección General de Enseñanza Superior.
} 


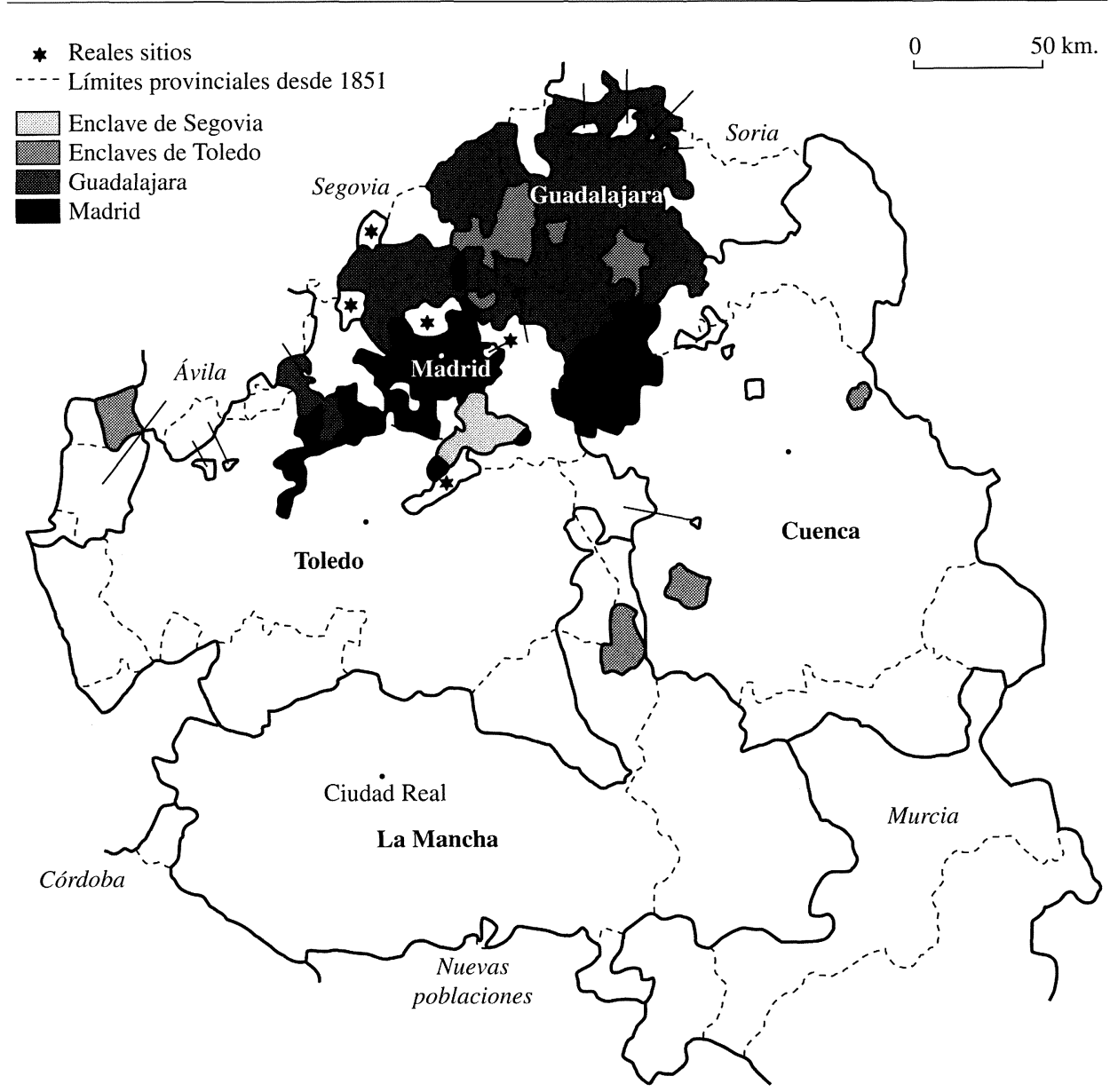

(Fuente básica: M. D. Mareas González, 1971).

Figura 1.-Las provincias en 1789.

- Reformas ilustradas impulsadas por el Consejo de Hacienda de 1799 a 1805, con importantes rectificaciones en los límites tradicionales (figura 2).

- División en prefecturas por el gobierno intruso de José I (1810) según el diseño de José de Lanz.

- Proyecto de división provincial del marino y cartógrafo mallorquín F. Bauzá (1813), cuyas principales novedades eran la supresión de la provincia de Guadalajara y la creación de una gobernación subalterna de Toledo en Talavera. 
- Proyecto de división provincial de Bauzá y del ingeniero de canales J. A. de Larramendi (1821): primer diseño de la provincia de Albacete y cesión de la capitalidad de Guadalajara a Brihuega.

- Modificación del proyecto por una comisión parlamentaria presidida por Diego Clemencín: restablecimiento de la capitalidad de Guadalajara y sustitución de Albacete por Chinchilla. Tras la discusión parlamentaria, la nueva división de España en 52 provincias fue sancionada por las Cortes el 27 de enero de 1822 (figura 3), siendo suprimida al final del Trienio liberal, al igual que las primeras divisiones en partidos judiciales.

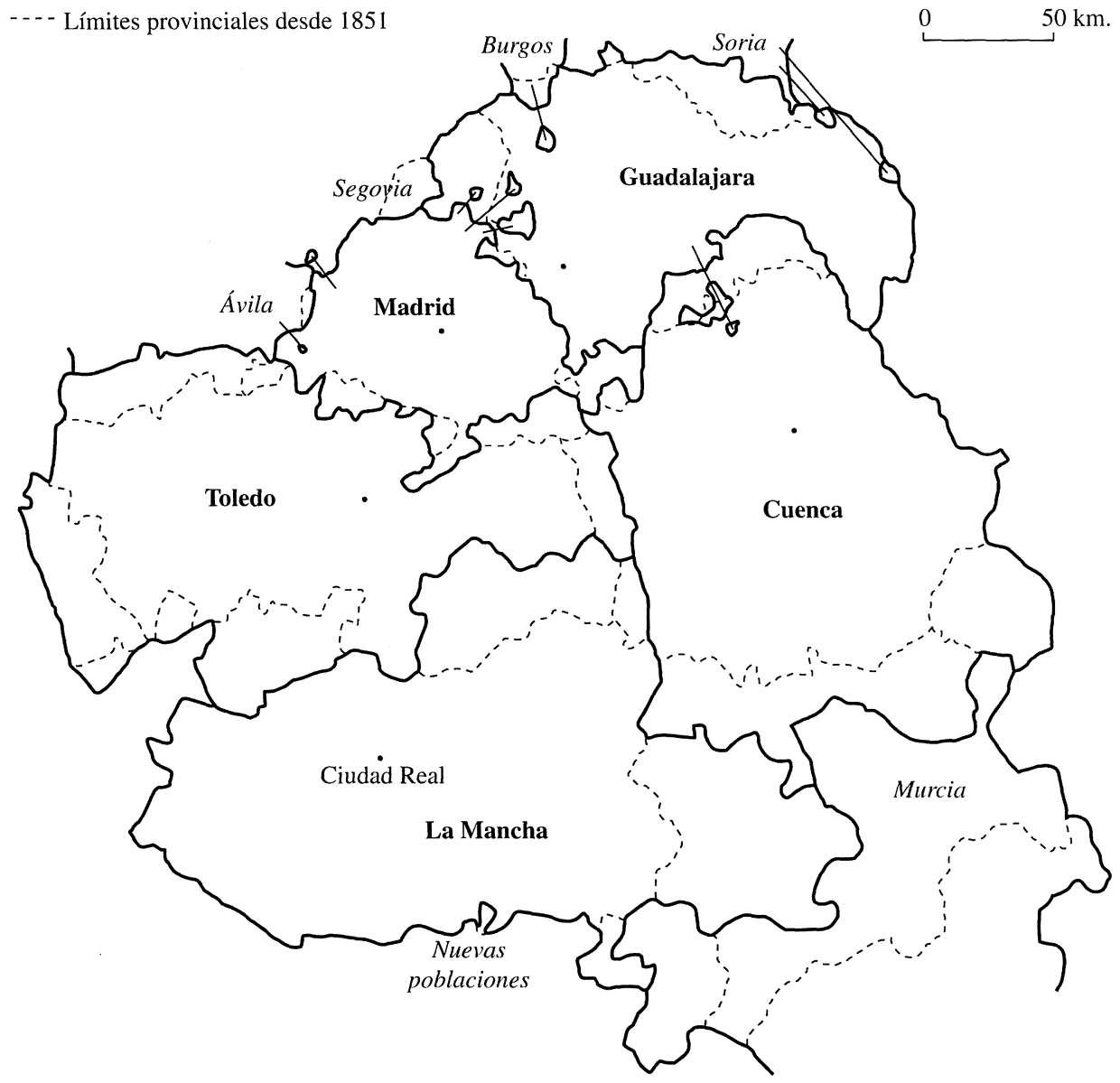

FiguRA 2.-Las reformas ilustradas (1799-1805). 


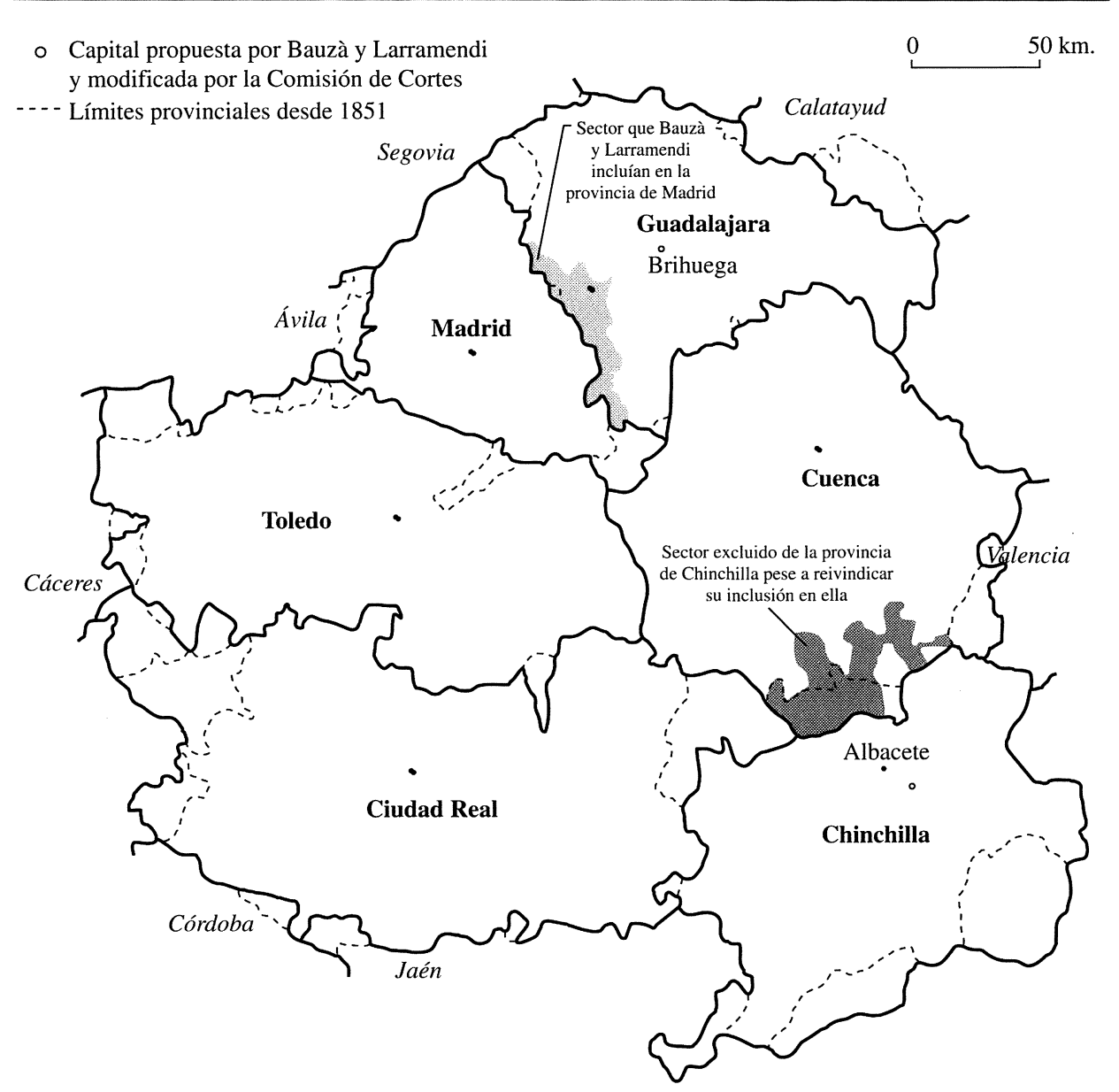

Figura 3.-Las provincias de 1822.

- Elaboración de un nuevo proyecto de división provincial por una comisión creada en 1825, en la que nuevamente tomó parte Larramendi: Albacete obtiene definitivamente la capitalidad. Por lo que se refiere a los límites, el proyecto es prácticamente un calco de la división aprobada en Cortes y así debe entenderse si no señalamos lo contrario; no obstante conviene destacar una importante diferencia: la repercusión del restablecimiento de los límites históricos castellanos con los antiguos reinos de la Corona de Aragón.

- En 1829 se trasladó la propuesta a las audiencias y a las chancillerías de Valladolid y Granada para su revisión, así como para el 
diseño de una nueva planta de partidos judiciales. La comisión central dio los últimos retoques a la división territorial en función de la información recopilada, y finalmente se aprobó mediante un decreto promovido por Javier de Burgos (30-XI-1833); otro tanto sucedió con el nuevo mapa de partidos judiciales (D. 24-IV-1834).

- En 1842 el geógrafo Fermín Caballero ultimó un plan para reformar la división provincial que no llegó a tener efecto ${ }^{1}$. Manzanares habría sustituido a Ciudad Real como capital.

- La última modificación territorial de Castilla-La Mancha se produjo en 1851, con la unión de Requena y Utiel a Valencia.

\section{Las reformas territoriales de 1799-1805}

En lo que atañe al mapa provincial castellano, las reformas ilustradas de principios de siglo constituyen el eslabón perdido en la evolución entre el caos territorial del Antiguo Régimen y la racionalidad de las divisiones liberales. El ciclo de reformas se inició a finales de 1799, con el cambio de provincia de algunos partidos: los hasta entonces distritos toledanos de Alcalá de Henares y Alcázar de San Juan pasaron, respectivamente, a Madrid y a La Mancha, al tiempo que el guadalajareño partido de Colmenar Viejo se agregaba a Madrid (R.O. 8-XII-1799). A estas disposiciones puntuales siguieron las reformas globales de cada provincia, realizadas entre 1801 y $1805^{2}$.

El resultado final de las reformas ilustradas resultó verdaderamente revolucionario (figura 2). La distancia a la capital se convirtió en el criterio prioritario en la demarcación provincial y, en consecuencia, los límites aparecen indudablemente más nítidos y regularizados. Igualmente queda claramente deslindada la Castilla meridional de la septentrional mediante la utilización del Sistema Central. Las ciudades de la Meseta norte pierden sus territorios de ultrapuertos: Segovia sólo logra retener el valle de Lozoya. El cúmulo de en-

1 Ya en 1822 Fermín Caballero habla tomado parte en tareas de reordenación territorial, proponiendo a la Diputación de Madrid la división de la provincia en ocho partidos judiciales (RAH: leg. 9-4680, fols. 164-167).

2 AHN: Hacienda, libro 6103, doc. 22 (Madrid, 31-I-1801) docs. 143 y 144 (Guadalajara y Toledo, 17-X-1801) y libro 6102, doc. 12 Cuenca, 13-II-1805). Ignoramos la fecha de incorporación de la localidad cordobesa de Chillón a La Mancha, anterior en todo caso a 1813. 
claves de unas provincias en otras se consigue reducir en gran medida: los que persisten se deben al descuido de los autores del plan o a la deficiencia de la cartografía (básicamente los mapas de Tomás López) y de la información estadística utilizada. Otras disfuncionalidades son imputables al modo de operar, traspasando la totalidad de algunas demarcaciones de una provincia a otra en función de la lejanía de su capital a las pocas ciudades que servían como referencia; así, si bien era admisible la unión de Alcázar de San Juan a Ciudad Real, diversos pueblos de su partido se hallaban en realidad más próximos a Toledo.

La hoy provincia de Madrid adquirió su perfil actual en 1801. Es en este momento cuando se incorporan a su demarcación el conglomerado de enclaves y cuñas de otras provincias que rodeaban a la corte: antiguos territorios de Guadalajara (Colmenar Viejo, San Martín de Valdeiglesias ${ }^{3}$, Galapagar...), de Segovia (Chinchón, Navalcarnero, Valdemorillo...), de Toledo (Alcalá de Henares, Valdemoro ${ }^{4}$, Fuente el Saz ${ }^{5} .$. ) y los reales sitios de Aranjuez, El Escorial, El Pardo y San Fernando de Henares. Al actual triángulo provincial únicamente le faltaban sus vértices: los valles del Lozoya (repartidos aún entre Segovia y Guadalajara), los pueblos comprendidos entre el Tajuña y el Tajo ${ }^{6} \mathrm{y}$ el sector de Cadalso (todos ellos en Toledo). Por contra, del territorio madrileño se segregó el apéndice internado en Toledo (Maqueda) y parte del enclave situado entre las provincias de Guadalajara y Cuenca (Pastrana, pero no Almonacid de Zorita). El diseño provincial se basaba en la comparación de las distancias a Madrid o Alcalá de Henares respecto de las otras capitales de partido próximas (Segovia, Toledo, Ocaña y Guadalajara), pero adoptaba también criterios de corte naturalista, como queda de manifiesto en esta alusión al Sistema Central:

«La villa de Santa María de la Alameda, de la provincia de Segovia, dista una legua y media menos de dicha ciudad que de Madrid, pero se ha agregado a esta provincia con los demás pueblos de aque-

3 San Martín había pertenecido, como Méntrida, Villa del Prado y La Torre de Esteban Hambrán, al partido de Colmenar Viejo (Guadalajara). La real cédula indica que todos ellos prefirieron unirse a Madrid en vez de a Toledo.

4 Juntamente con Esquivias, que solicito su unión a Madrid.

5 Cabanillas de la Sierra y Redueña quisieron estar en Madrid. No así Torrelaguna, que optó por incorporarse a Guadalajara.

${ }_{6}$ El pueblo segoviano de Villaconejos solicité estar en el partido de Ocaña (Toledo); por contra, el toledano Perales de Tajuña opto por unirse a Madrid.

$$
-360-
$$


lla que quedan expresados por hallarse todos situados de puertos acá, y pagar aquí desde antes del nuevo plan sus encabezamientos de contribuciones por gracia que se les dispensó.»

También la provincia de Guadalajara es en gran medida una creación ilustrada o preliberal. Con anterioridad a la reforma de 1801 la mitad de su territorio actual dependía de otras cinco ciudades castellanas: Cuenca (Señorío de Molina), Soria (Atienza y el Ducado de Medinaceli), Toledo (Brihuega y otros enclaves), Madrid (Pastrana) y Segovia. En esta ocasión se agregaron todos los territorios que -según los mapas utilizados- se encontraban más cercanos a Guadalajara o Sigüenza, las dos capitales de partido en el ramo de Hacienda. Además de este criterio principal, también en la concepción del territorio guadalajareño puede observarse una orientación naturalista, pues su demarcación se corresponde -esencialmente con las tierras tributarias del Tajo por su derecha. En el mismo sentido, como límite entre Toledo y Ávila se adoptó la alineación de cumbres de la sierra de Gredos, a medio camino de las ciudades de referencia: las dos capitales provinciales y Talavera de la Reina ${ }^{7}$. Esto comportaba la inclusión en Toledo de los antiguos partidos de Oropesa, Mombeltrán y Navamorcuende ${ }^{8}$.

La reordenación de los límites también fue importante en el área de contacto de las provincias de Cuenca, La Mancha y Toledo. El trasvase de mayor magnitud consistió en la agregación a La Mancha del antiguo partido toledano de Alcázar de San Juan, internado profundamente en el territorio de aquella provincia. Otros muchos municipios pasaron de unas provincias a otras (Villarrobledo de La Mancha a Cuenca ${ }^{9}$, Mota del Cuervo de Toledo a La Mancha, Saelices de Toledo a Cuenca...), al tiempo que desaparecían todos los enclaves en esta zona.

La mayor parte de las reformas concluidas en 1805 persistieron hasta 1833, salvo el lapso de año y medio de vigencia de la división

\footnotetext{
7 Peraleda de la Mata (no confundir con la toledana Peraleda de Garvín o de San Román) se unió a Talavera de la Reina, pues prefería esta adscripción a la de Plasencia.

8 Que sepamos, únicamente las poblaciones del alto Tiétar (sector de La Adrada) protestaron por este cambio. No se aceptó su reintegración a Ávila, e incluso se enmendó el olvido de Casillas y Gavilanes en el listado original, uniéndolos también a Toledo (AHN: Reales Cédulas, n. 1409, 24-III-1802).

$9 \mathrm{Al}$ igual que Balazote y Manera, que así lo solicitaron por ser el camino a San Clemente -cabeza de partido- más llano y seguro.
}

$$
\text { - } 361 \text { - }
$$


aprobada en Cortes. No obstante, hay que anotar la reintegración a Ávila de sus antiguos límites por real orden de 22 de mayo de $1820^{10}$. Pese a la importancia de la reestructuración territorial de principios de siglo, el hecho de no haber sido plasmada cartográficamente comportó que fuese, si no desconocida, sí insuficientemente asumida por sus contemporáneos. La imagen de la distribución provincial castellana seguía siendo la que transmitían los mapas de Tomás López elaborados en la segunda mitad del siglo xviII; la percepción de caos territorial no desapareció, por tanto, en quienes afrontaron la gestación de una nueva organización provincial. Un ejemplo lo hallamos en la intervención en Cortes del diputado manchego Ramón Giraldo: «una de las causas de los males que experimenta la provincia de La Mancha es esa misma confusión de pueblos y de límites, mezclándose con los de las provincias de Cuenca, Toledo y Murcia; y habiendo pueblos como Los Hinojos y Yébenes que pertenecen a dos, debería adoptar, aunque el provincialismo me saliese a borbotones, cualquiera división que demarcase con más claridad cada provincia, y que facilitase todos los medios que ha de tener para su fomento y felicidad» ${ }^{11}$.

\section{Las propuestas de Lanz (1810) y Bauzà (1813)}

La división decretada por José I mantenía las cinco capitales tradicionales de Castilla la Nueva, pero alteraba radicalmente los límites de sus respectivas prefecturas. Por lo que respecta a los confines periféricos hay que destacar la utilización de la totalidad del Sistema Central como divisoria entre ambas Castillas, así como la segregación de Molina para Zaragoza, Talavera para Cáceres, Horcajo de los Montes para Badajoz y Requena para Valencia. Merece también rese-

10 Listado del repartimiento de 1821 (DSC: 8-III 1821, p. 286), en el cual también se constata que Bustarviejo y Valdemanco permanecieron en Segovia pese a haber sido incluidos en Guadalajara en 1801. En otro orden de cosas, al aprobarse la división de partidos de Guadalajara (O. 12-VII-1820) se incorporaron a esta provincia los pueblos de Villel de Mesa y La Yunta, agregados a Soria en 1801, así como la localidad conquense de Carrascosa, situada al norte del Tajo.

11 DSC: 5-X-1821, p. 118. El actual pueblo de Los Hinojosos estaba formado por Hinojoso de la Orden (La Mancha) e Hinojoso del Marquesado (Cuenca), obviamente de jurisdicciones diferentes. En el Diario de Sesiones de 25-V-1821 figura su solicitud de 
ñarse el desplazamiento del límite meridional de Guadalajara hasta la divisoria de vertientes del Tajo al Júcar y al Guadiana, de tal modo que la provincia de Cuenca quedaba sensiblemente reducida y su capital en una posición netamente excéntrica.

Lo que más llama la atención del diseño departamental en la Castilla meridional es la reducción de Madrid a un distrito que hoy calificaríamos de metropolitano, puesto que su demarcación no alcanzaba más allá de Alcalá de Henares por el Este, en tanto que poblaciones como Colmenar Viejo, El Escorial, Navalcarnero, Aranjuez o Chinchón quedaban adscritas a Toledo. Como señala Francisco García Martín (1988, p. 398), la reducida extensión de la prefectura madrileña (unos $2.300 \mathrm{~km}^{2}$ ) evoca la solución adoptada en la configuración del departamento de París. Este diseño excepcionalista de la prefectura de la capital del Estado fue sensatamente descartado en todos los ensayos posteriores, de tal modo que Madrid adquirió un territorio mucho más acorde con su incontrovertible primacía en la jerarquía urbana.

En su proyecto de 1813, Felip Bauzá demuestra ignorar totalmente las reformas emprendidas en tierras castellanas a principios de siglo, pues propone un reparto de la provincia de Guadalajara según las directrices de la demarcación dieciochesca: el Señorío de Molina se agregaba a Cuenca, el Ducado de Medinaceli a Soria y la antigua pequeña provincia guadalajareño a Madrid. La otra gran novedad del plan de Bauzá era la creación de una gobernación subalterna en Talavera de la Reina, posibilidad que ya no se contemplaría en 1821, pese a la petición que en este sentido cursó su Ayuntamiento ${ }^{12}$. En el resto de gobernaciones efectuaba una simple regularización de los límites antiguos, aunque mostrando especial predilección por los cursos fluviales, especialmente el Tajo y el Záncara. En cuanto a los confines periféricos de Castilla-La Mancha debe señalarse la reiterada adscripción de Requena y Utiel a Valencia y, en sentido contrario, la incorporación a La Mancha del sector extremeño de Herrera del Du-

unión bajo el nombre de Hinojoso de la Constitución. Por lo que respecta a Los Yébenes, el diputado yerra, pues tanto Yébenes de San Juan como Yébenes de Toledo se hallaban en la provincia toledana.

12 Archivo del Congreso de Diputados, en adelante AC: leg. 78, n. 74, lista de peticiones de 20-VIII-1820. 
que y Puebla de Alcocer ${ }^{13}$, así como a la gobernación subalterna de Talavera de parte de la vertiente meridional de la sierra de Gredos (aunque sorprende la unión de Arenas de San Pedro y Candeleda a Salamanca).

Con todo, más que estos precedentes de la Guerra de la Independencia, sería el proyecto ultimado en 1821 por Bauza en unión de Larramendi el que marcaría las grandes líneas del diseño provincial definitivo en Castilla la Nueva. En los apartados siguientes centraremos nuestra atención en este plan, en su discusión parlamentaria y sus avatares posteriores.

\section{La nueva provincia manchega}

La nueva provincia manchega -a caballo de Castilla (Cuenca y La Mancha) y del antiguo reino de Murcia- no aparece definida hasta la intervención de José Agustín de Larramendi en el proyecto de división territorial. El ingeniero de canales conocía bien estas tierras, pues a principios de siglo tomó parte en los trabajos de desecación de las lagunas próximas a Albacete (Larramendi, 185859). Esta ciudad aparece designada como capital tanto en el proyecto que elaboró juntamente con Bauzá en 1821 como en el que se hizo público en 1829. Sin embargo, la comisión de Cortes presidida por el murciano Clemencín se decantó a favor de Chinchilla como capital de la Mancha Alta, en atención a los siguientes argumentos: "La comisión ha vacilado al asignar su capital entre los pueblos de Albacete y Chinchilla. A favor del primero hay la circunstancia de estar colocado en la reunión de las carreteras para Valencia y Cartagena, su situación en una llanura fácilmente accesible de todas partes, su mayor población, celebridad y concurrencia de su feria. A favor del segundo está su mayor salubridad, la calidad de cabeza de partido declarada por las Cortes en competencia con Albacete, el título de ciudad a que va aneja su mayor importancia en lo antiguo y el ser residencia y asamblea de un regimiento provincial que lleva su nombre, sin carecer de edificios,

13 En la interpretación de las indicaciones de Bauzá deben tenerse presentes los mapas de Tomás López, y concretamente el curso del Guadalemar, afluente del Zújar que López dibuja como tributario directo del Guadiana. 
caminos y demás circunstancias convenientes para la capitalidad. En vista de todo la comisión se inclina a Chinchilla.» ${ }^{14}$ Semejante elección estuvo influenciada, sin duda, por el hecho que el ministro de Justicia, Vicente Cano Manuel, y su hermano Antonio (diputado a Cortes por Murcia) fuesen naturales de Chinchilla.

En este contencioso resultaba irrelevante disputar acerca de la mayor centralidad de una u otra población, distantes entre sí apenas 13 kilómetros; por consiguiente el debate tuvo que centrarse en aspectos urbanisticos, topográficos y ambientales. El valenciano Antoni Bernabeu, tras realizar una completa descripción geográfica de ambas ciudades, observa que la situación de Chinchilla en el alto de un cerro hacía «muy difícil o imposible que se extienda la población, como probablemente es de esperar suceda en lo sucesivo a todas las capitales de provincia» ${ }^{15}$. A ello oponían los partidarios de Chinchilla el valor estratégico de su emplazamiento, lo que a su vez era impugnado por el aragonés Ceferino Lagrava: «no serán la cuesta y tapias de Chinchilla las que garantizarán la seguridad de aquellas autoridades» ${ }^{16}$. La ubicación topográfica tenía además una relación directa con la salubridad. En la cuenca endorreica donde se halla Albacete era normal el estancamiento de las aguas, causa de enfermedades y tercianas. Los partidarios de Chinchilla hicieron especial hincapié en estas preocupaciones higienistas; Antonio Cano realizó una tediosa loa de su ciudad natal salpicada con afirmaciones de este tipo: «Abundan además en su suelo yerbas y arbustos aromáticos que impregnan el aire, que es el que constituye su salubridad, don de la naturaleza que no tiene Albacete» ${ }^{17}$. Por contra, los que defendían la propuesta de Bauza y Larramendi señalaban que el canal construido en 1805 había paliado en gran parte la problemática de las lagunas y buscaban la parte positiva de este fenómeno natural, cual era la abundancia de aguas, de las que -según ellos- carecía Chinchilla. En cuanto a la posesión del título de ciudad que se menciona en la justi-

14 DSC: $1-\mathrm{X}-1821$, p. 57.

15 DSC: $11-\mathrm{X}-1821$, p. 200

6 DSC: $12-\mathrm{X}-1821$, p. 209

17 DSC: 13-X-1821, p. 225. En mayo de 1830, Antonio Cano Manuel también abogó a favor de Chinchilla ante el magistrado Lamas Pardo, responsable de supervisar los proyectos de partidos judiciales, (Berazaluce, 1967, p. 207); por entonces el político manchego presidía la junta directiva del canal de Albacete, luego llamado de María Cristina. 
ficación de la comisión de Cortes, el valenciano Antoni Verdú opuso, en una concisa y aguda intervención, que «por ciudad no debía ser Madrid la corte, porque no lo es, debería serlo Guadalajara» ${ }^{18}$.

No es fácil conocer con exactitud cuál de las dos ciudades era más populosa ${ }^{19}$, pero sí parece claro que el dinamismo económico era superior en Albacete, tal y como reconocía implícitamente Cano Manuel: «Albacete ha llegado al colmo de la felicidad que puede disfrutar: tiene un camino que no se le quita, tiene una gran feria que antes no tenía. La empresa de aguas, una vez reparados los males que yo he indicado, puede producir grandes beneficios al pueblo. ¿Qué es, pues, lo que se le quita con nombrar por capital a Chinchilla? [...] Las Cortes han propendido siempre, siguiendo el espíritu de la Constitución, a amparar al débil en competencia del poderoso» ${ }^{20}$.

La candidatura albaceteña era igualmente apoyada por la Diputación de Murcia y el jefe político ${ }^{21}$. La postura de la corporación provincial resulta un tanto sorprendente, pues apenas un año atrás había propuesto como capital de partido a Chinchilla, y no a Albacete. $\mathrm{Al}$ parecer el nuevo posicionamiento se debió a un enrarecimiento de las relaciones con el Ayuntamiento de Chinchilla, a causa de su actitud obstruccionista ante las segregaciones municipales promovidas en su extenso término. La Diputación murciana llegaba a acusar a esa corporación municipal de actuar en ese tema, "como en todo», de forma ajena al espíritu de la Constitución ${ }^{22}$. La situación llegó a tal punto de deterioro que el jefe político de Murcia impuso el traslado

18 DSC: $13-\mathrm{X}-1821$, p. 230.

19 Miñano concede una ligera ventaja a Chinchilla (10.533 habitantes frente a 9.074 de Albacete), pero los datos facilitados por el Ayuntamiento de Albacete en 1829 son radicalmente opuestos: 10.860 para esta ciudad y sólo 5.376 para Chinchilla. Las cifras empleadas en 1834 son algo más equilibradas: Albacete tendría 11.202 habitantes y su oponente 7.060. En el censo de 1857 Chinchilla figura como la sexta ciudad de la provincia (6.044 habitantes), muy por debajo de Albacete (16.607)

20 DSC: $13-\mathrm{X}-1821$, p. 228.

21 Archivo Regional de Murcia, en adelante ARM: Actas de la Diputación, vol. III, fol. 157, 9-IV-1821 y DSC: 6-VI-1821.

22 El enfrentamiento llegó al punto de ser la Diputación quien animara a los vecinos de Pétrola y Villar a unirse para formar ayuntamiento segregado, «quedando libres del influjo de los grandes propietarios de Chinchilla» (ARM: Actas de la Diputación, vol. III, 7-VI-1821). Contrariamente, Antonio Cano declaraba que Chinchilla había ac- 
del juzgado de Chinchilla a Albacete, como castigo por haber desobedecido su autoridad ${ }^{23}$.

Los ayuntamientos de la provincia se decantaban claramente a favor de Albacete, a la luz de los numerosos pronunciamentos en uno $\mathrm{u}$ otro sentido registrados en el Diario de Sesiones. Albacete conseguía, además, el apoyo de las principales poblaciones: Almansa, El Bonillo, Casas-Ibáñez, Hellín, Jumilla, La Roda, Tarazona, Tobarra... Pese a todo, la mayoría del Congreso apoyó finalmente el dictamen de la comisión parlamentaria, siendo designada Chinchilla como capital. En 1829 cambiaron las tornas, y se impuso el criterio de Larramendi. Chinchilla vería peligrar incluso su capitalidad de partido judicial, pues el proyecto que remitió el Ayuntamiento de Albacete a la Chancillería de Granada no la contemplaba como tal (Ñacle: 1990, p. 148).

La división del antiguo Reino de Murcia no suscitó en modo alguno reticencias por parte de los diputados de esta provincia. Al contrario, este grupo de parlamentarios se significó por su apoyo al proyecto de la comisión. El deslinde de ambas provincias tampoco suscitó grandes problemas. La demarcación de 1822, en comparación con la actual, se hallaba desplazada hacia el Sur, puesto que se incluían en Chinchilla los municipios de Jumilla, Yecla, Moratalla ${ }^{24}$, Santiago de la Espada, Albadalejo y Terrinches, excluyendo en cambio otros como Ossa de Montiel, Villarrobledo, La Roda o Tarazona de la Mancha. Esta delimitación suscitó la protesta de numerosos pueblos conquenses que también querían ser incorporados a la nueva provincia (figura 3) ${ }^{25}$. Tal vez por esto Larramendi desplazó la demarcación albaceteña hacia el Norte, situándola en su trazado actual, excluyendo por el Sur a Moratalla y Jumilla y agregando Villena y Sax. Esta versión aún sufrió alguna que otra enmienda antes y después de 1833: en 1829 la Chancillería de Gra-

tuado en este contencioso como el "padre que ha establecido una familia numerosa» (DSC: 13-X-1821, p. 229).

23 AC: leg. 37, exp. 62. Josep Fontana (1983, p. 159) alude a otra vertiente de este conflicto.

${ }_{24}$ Moratalla manifestó reiteradamente su deseo de reintegrarse a Murcia, a lo que se oponía la Diputación de Chinchilla (ARM: Actas, vol. V, 24-V y 7-VIII-1822). El diputado La-Santa era partidario de adoptar como límite septentrional de Murcia la divisoria de los ríos Argos y Quipar, de tal modo que también serían agregados a la provincia manchega Calasparra, Caravaca y Cehegín (DSC: $21-\mathrm{X}-1821$, p. 333).

25 DSC: 26-X, 15, 18 у 25-XI-1821. 
nada ya contemplaba la incorporación de Ossa de Montiel, en tanto que el Ayuntamiento de Ciudad Real proponía dejar Villarrobledo en su provincia; en 1831 se trabaja con la hipótesis de dejar Santiago de la Espada en Jaén ${ }^{26}$ y en el decreto de Javier de Burgos se dejan Yecla y Sax para Murcia, y Albadalejo y Terrinches en Ciudad Real. En 1836 Villena fue unida a Alicante y, finalmente, el 24 de marzo de $1846 \mathrm{~J}$. de Burgos -nuevamente ministro de la Gobernación- ordenó la incorporación a Albacete de Villarrobledo, cuyo Ayuntamiento se quejaba de la inseguridad del camino que conducía a la lejana Ciudad Real.

Pese a la progresiva ampliación territorial albaceteña hacia el Norte, es evidente que la capacidad de atracción de esta ciudad desbordó desde el primer momento (y con mayor intensidad lo hace hoy día) la demarcación provincial septentrional. En relación a esta cuestión, resulta significativo observar que tampoco el conquense Fermín Caballero estaba libre del pecado del provincialismo, puesto que en 1863 preparó para el gobernador de Cuenca un informe contrario al trasvase de más municipios de esta provincia a Albacete (López Gómez: 1978, p. 43).

\section{El concepto de centralidad en la provincia de Guadalajara}

Bauzá y Larramendi opinaban que Guadalajara (6.736 habitantes según Miñano) no podía «en ninguna manera quedar cabeza de provincia y distante sólo ocho leguas de Madrid», por lo que la unían a la provincia de la corte; en su lugar señalaban como capital a Brihuega (4.422 hab.), aunque admitían la posibilidad que lo fuera Cifuentes (1.394 hab.). También Sigüenza aspiraba a conseguir la capitalidad, en tanto que sede episcopal y segunda población de la provincia: 4.868 habitantes ${ }^{27}$. La comisión de Cortes, tras consultar la cuestión con los diputados guadalajareños, decidió conservar la antigua capital y desplazar un tanto los límites hacia el Oeste, prácticamente a su trazado definitivo (figura 3). La elección de Guadalajara se justificaba

26 Biblioteca Nacional: Ms. 1907 y 1909.

27 En el Diario de Sesiones constan las solicitudes de Sigüenza (27-VIII-1820), la réplica de Guadalajara (20-IX-1820) y las representaciones de Cifuentes (26-IV-1821) y Brihuega (6-IV y 10-X-1821). Véase el estudio de Pedro Ortego (1990), particularmente pp. 899-900. 
del modo siguiente: «ha sido [capital] hasta ahora, y está situada del modo más ventajoso para la pronta comunicación con el gobierno supremo y con los pueblos de la provincia» 28 .

El debate por la capitalidad de las tierras del alto Tajo ejemplifica la confrontación entre dos concepciones de la centralidad: geométrica o absoluta por un lado y ponderada o relativa por otro. Brihuega y Cifuentes representan la opción por la centralidad geométrica defendida por los comisionados del Gobierno y, en el debate parlamentario, por los diputados Pedro Juan de Priego, Romero Alpuente y Marcial López: ninguno de ellos guadalajareño. Así, Marcial López se pregunta: « $i$ a quién se le ofrece poner la capital de una provincia en su misma línea divisoria casi, es decir, legua y media de los términos de otra, en un límite que dista de otro más de 30 leguas, y casi en las mismas puertas de Madrid?» ${ }^{29}$. Los partidarios de Brihuega señalaban, además, la importancia de su mercado semanal y del establecimiento nacional de paños.

Por su parte, los diputados favorables a Guadalajara remarcaban la importancia industrial y comercial de esta ciudad, su mayor accesibilidad y centralidad ponderada, así como la conveniencia de mantener la capital histórica: «La centralidad que ha considerado y considera la comisión está, no precisamente en el centro material del territorio, sino en el de la población y del movimiento, y en este concepto yo quisiera que se me dijese si Guadalajara no está en la parte más poblada y de mayor movimiento industrial de la provincia. [...] Las comunicaciones antiguas de la provincia, los caminos buenos o malos que hay de travesía, van a parar a Guadalajara, a donde los pueblos están habituados a acudir hace siglos, y la fuerza de las habitudes es tal, que aun no siendo enteramente agradables, suele incomodarnos que se interrumpan» ${ }^{30}$.

Al fin y al cabo, tal y como apuntaba el guadalajareño Anselmo A. Fernández, esta ciudad es la puerta de entrada a la verdadera capital funcional, que no es otra que Madrid: «La verdad es que los que habitan el Señorío de Molina tienen su verdadero interés en tener a Guadalajara por capital, y no a Cifuentes, Brihuega ni otro pueblo,

28 DSC: $1-\mathrm{X}-1821$, p. 57.

29 DSC: 10-X-1821, p. 176.

30 Clemencín, en DSC: 10-X-1821, p. 177. 
porque sus relaciones comerciales están en Madrid por Guadalajara» ${ }^{31}$.

Finalmente las Cortes refrendaron el dictamen de la comisión parlamentaria. Debe observarse que el descarte de la capitalidad de Brihuega no supuso la reintegración a Cuenca de los pueblos situados al sur del Tajo, unidos a su nueva provincia en razón de su cercanía a la localidad alcarreña ${ }^{32}$. Con todo, si la provincia de Guadalajara se ensanchó hacia el Sur fue también a costa de un retroceso por el Norte en función de la divisoria de aguas del Tajo al Duero y Jalón ${ }^{33}$.

La otra gran cuestión relacionada con la definición territorial de la provincia de Guadalajara fue la incorporación del antiguo Señorío de Molina. Este corregimiento tuvo una problemática inserción provincial a causa de su ubicación equidistante a las capitales cercanas. Hasta 1801 perteneció a la provincia de Cuenca, y desde entonces a la de Guadalajara; Bauzá y Larramendi cedieron a la provincia de Calatayud los pueblos situados en la cuenca del Jalón ${ }^{34}$, y Fermín Caballero ampliaba esta agregación a la totalidad del partido de Molina. El contencioso sobre la identidad territorial del Señorío ya se había puesto de relieve en la primera etapa constitucional. El hecho de haber contado con una Junta propia en la Guerra de Independencia permitió a Molina tener dos diputados a Cortes y, gracias a esto, reivindicar la categoría de provincia ${ }^{35}$. Ya en la discusión del artículo décimo de la Constitución, que hace referencia a los territorios de las Españas, el canónigo molinés José Roa logró que se añadiera el Señorío de Molina a la enumeración de los antiguos reinos y provincias ${ }^{36}$. El asunto no tenía, desde luego, un valor testimonial sino que

31 DSC: 10-X-1821, p. 179.

32 La protesta de la Diputación de Cuenca figura en AC: leg. 39, n. 85, 17-VI-1822.

33 En este confín la descripción de 1822 registra algunas lagunas o contradicciones: Barcones y Marazovel quedaron en tierra de nadie, excluidos tanto de Guadalajara como de Soria. Barcones solicitó permanecer en Guadalajara: Ortego (1990, p. 904). El deslinde de ambas provincias seguía siendo problemático en el decreto de 1833: Madrigal y Paredes de Sigüenza quedaban en Soria y no se aclaraba la ubicación de Iruecha; la inmediata subdivisión en partidos judiciales solventó estas ambigüedades.

34 El Ayuntamiento de Molina se quejó de la desmenbración de su partido (DSC: 13-X-1821, p. 223).

35 Acerca de las irregulares circunstancias que permitieron al Señorío de Molina contar con representación parlamentaria en Cádiz véas el estudio de Pilar Chávarri (1988, pp. 316-327).

36 DSC: 2-IX-1811, pp. 1742-1744. 
buscaba crear un precedente que permitiera la posterior consecución de diputación provincial propia, tal y como reclamó Ramón López Pelegrín ${ }^{37}$. No obstante, en esta ocasión lo único que concedieron las Cortes fue que la denominación provincial fuese la de «Guadalajara con Molina». La reivindicación secesionista parece ya olvidada en el Trienio, pese a que el aragonés Marcial López apuntase la posibilidad de formar una nueva provincia de Molina ${ }^{38}$.

\section{La polémica capitalidad de Ciudad Real}

También en la antigua provincia de La Mancha se produjo cierta pugna por la capitalidad entre Ciudad Real, Almagro y Manzanares, todas ellas con una población prácticamente idéntica: 10.200 habitantes en $1857^{39}$. Sin embargo, no fue en las Cortes de 1821 cuando esta disputa tuvo mayor relevancia, sino en las constituyentes de 1837. Almagro (que ya había sido sede de la Intendencia manchega entre 1750 y 1761, y capital del extenso partido de la orden de Calatrava hasta su reunión al corregimiento de Ciudad Real a principios de siglo) contaba con el apoyo de diversos pueblos de la provincia ${ }^{40}$. No obstante, en 1842 Fermín Caballero se decantaba a favor de Manzanares, sin que conozcamos las razones que le inducían a preferir esta ciudad, la más excéntrica de entre las que estaban en liza ${ }^{41}$. Con todo, las iniciativas encaminadas a desplazar la capitalidad de Ciudad Real no prosperaron.

Por lo que se refiere a los límites de la provincia de Ciudad Real resulta llamativo el flujo y reflujo que experimentó en su sector occi-

37 Pelegrín aceptaba, no obstante, que Molina continuase adscrita a la Intendencia de Guadalajara y a esta circunscripción electoral (DSC: 3-V-1812, pp. 3133-3138). El también molinés y diputado por Guadalajara, Andrés Esteban, se opuso a esta petición.

38 DSC: $10-\mathrm{X}-1821$, p. 177.

39 En cambio, según datos de la división en partidos judiciales de 1834 (que Fermín Caballero reproduce en una de sus colaboraciones en el Eco del Comercio de ese mismo año), Almagro alcanzaba los 14.760 habitantes, en tanto que Manzanares y Ciudad Real contaban con poco más de 10.700 .

40 DSC: $11,13,14$ y 17-II-1837; y 6 y 9 -III-1837. Puede verse un alegato de Ciudad Real en defensa de su capitalidad, y en oposición a Almagro, en Pillet: 1984, pp. 173-184.

41 Manzanares lo había solicitado en el Trienio (AC: leg. 78, n. 74, lista de peticiones de abril de 1821) y en 1837 (DSC: 17-II- 1837). Curiosamente, según F. Caballero, también fue una comisión de la que él formaba parte la que en 1834 decidió dar la ca- 
dental entre $1822^{42}$ y 1833 . No es, desde luego, casual que se tratara de una zona mal conocida y pésimamente plasmada cartográficamente. En la división constitucional la provincia de Ciudad Real se extendió hasta comprender un vasto sector de los Montes de Toledo y diversas poblaciones anteriormente extremeñas, como Herrera del Duque y Capilla (figura 3). La intervención de la Audiencia de Extremadura en 1829 fue decisiva -como se deduce de la documentación divulgada por Muñoz de San Pedro- para que todos los pueblos extremeños que el plan gubernamental ${ }^{43}$ volvía a adscribir a La Mancha permanecieran finalmente en Badajoz, lo cual indirectamente convirtió Anchuras en enclave.

\section{Los limites de Toledo}

Posiblemente el límite más polémico de Castilla-La Mancha fue el de Toledo con Cáceres y Ávila. Anteriormente hemos comentado que la reforma de 1801 incorporó a Toledo todos los territorios abulenses de ultrapuertos, pero que en 1820 Ávila consiguió recobrar su antigua demarcación, con el dilatado enclave de Oropesa. Al señalar el curso del Tiétar como línea divisoria, Bauzá y Larramendi apuntaban una solución intermedia, de modo que se agregaban a Toledo los sectores de Oropesa, Navamorcuende y Fresnedilla, pero conservando para Ávila la vertiente meridional de la sierra de Gredos con la adición de Arenas de San Pedro, anteriormente toledana; por otra parte, Cáceres adquiría el área de La Calzada de Oropesa. La comisión de Cortes determinó que la comarca de Arenas y Candelada se incluyera también en Toledo, pero no accedió a la proposición de los diputados toledanos Simón González Yuste, José de Manzanilla y Vicente de

pitalidad de un partido judicial a Manzanares, corroborando la propuesta de la Chancillería de Granada y en contra de lo dispuesto por el magistrado Lamas Pardo (Caballero, 1834).

42 Hay una lista de pueblos de Cuenca, Ciudad Real y Guadalajara referida a 1822 en el Archivo General de Simancas: Dir. Gral. del Tesoro, inv. 29, leg. 44. La correspondiente a Ciudad Real presenta algunas discrepancias de interpretación de los límites provinciales con los que cartografiamos: excluye Ossa de Montiel y Munera e incorpora Hontanar.

43 Aparte del sector de Villarrobledo, la única diferencia entre la división de 1822 y el plan de 1829 por lo que se refiere a Ciudad Real era la adscripción de la nueva población de Almuradiel a la provincia manchega en lugar de a Jaén.

$$
-372-
$$


La-Llave en el sentido de incorporar también el valle de Ramacastañas (Mombeltrán), que consideraban mal comunicado con Ávila a través del «fatal puerto del Pico o El Herrador, escabrosísimos, en extremo temibles, y que una gran parte del año se cierran absolutamente por la excesiva copia de nieves, en que han sido sepultados tantos infelices» ${ }^{44}$. Esta propuesta fue contrarrestada por otra de los diputados abulenses Eugenio de Tapia y Antonio de la Cuesta -con el apoyo de Agustí de Torres- quienes reclamaron para Ávila todas las poblaciones de la derecha del Tiétar hasta Candeleda, tal y como se dictaminó en 1833 tras las consultas a las audiencias y chancillerías.

Los antedichos diputados toledanos también elevaron otra propuesta que sí fue aceptada por las Cortes en 1822, referida a la incorporación a Toledo de diversas localidades que habían formado parte del antiguo partido abulense de Oropesa: La Calzada, Caleruela, Herreruela y Las Ventas de San Julián. Esa agregación venía a compensar la cesión a Cáceres de localidades tradicionalmente toledanas como Guadalupe o Castañar de Ibor ${ }^{45}$. Contradiciendo el plan de 1829 -idéntico a la división de 1822 por lo que se refiere a los límites provinciales toledanos- a la vista del informe de la Audiencia extremeña se determinó la incorporación a las provincias de Cáceres y Badajoz de un nuevo y nutrido grupo de localidades antiguamente toledanas: Valdecaballeros, Alía, Villar de Pedroso...

En el capítulo de proyectos fallidos de modificación de la división provincial toledana cabe reseñar la pretensión de la Chancillería de Granada de incluir en la provincia de Ciudad Real la comarca de Lillo en 1829 46, así como la intención de Fermín Caballero de agregar Quintanar de la Orden a Cuenca en 1842.

\section{La conformación de la provincia de Madrid}

La provincia que experimentó un mayor número de rectificaciones en los sucesivos proyectos fue la de Madrid. Si asimilamos su

${ }^{4}$ DSC: 20-X-1821, p. 316.

45 La primera división en partidos judiciales de Extremadura (DSC: 11-IX-1813) incluye diversos pueblos pertenecientes a Toledo (Alía, Bohonal de Ibor, Castilblanco, Guadalupe y Valdecaballeros) sin que conozcamos motivo para ello.

46 Biblioteca Nacional: Ms. 1909. 
forma a un triángulo, podríamos decir que las zonas de definición más problemática fueron sus tres vértices, en tanto que los tramos centrales de los lados de contacto con Guadalajara, Toledo y Segovia quedaron prácticamente determinados en 1821, una vez que la comisión de Cortes decidió la exclusión de la ciudad de Guadalajara de la provincia de Madrid. Así, si la adopción de la línea de cumbres del Guadarrama se mantiene invariable desde el primer momento, lo que no resultó tan sencillo de determinar fue el trazado de la línea que debía atravesar el Alberche separando Madrid de Ávila, al igual que la extensión de la cabecera del Lozoya que debía agregarse a Madrid. En ambos casos el trazado actual no se definió hasta la última fase de revisión del proyecto decretado por Javier de Burgos y, por lo que respecta al límite suroccidental, todavía en 1842 Fermín Caballero preveía agregar San Martín de Valdeiglesias a Ávila. En las dos zonas se observa una progresiva ampliación del territorio madrileño, que por lo que se refiere al Lozoya (en 1821 comprendía hasta Buitrago, en 1829 se extiende hasta Montejo y en 1833 se adopta la demarcación vigente) puede relacionarse con una política de incorporación de la cuenca hidrográfica destinada a abastecer de agua a la corte: cuestión en absoluto ajena a las preocupaciones de Larramendi.

El tercer vértice provincial en discusión, el suroriental, correspondía a una zona aproximadamente equidistante a las cuatro capitales próximas, y en la cual Madrid tuvo siempre alguna pertenencia. Aquí la evolución fue la contraria, ya que la demarcación madrileña se vio progresivamente recortada: en el siglo xviII Madrid contaba con el dilatado enclave de Almonacid de Zorita; en el primer tercio del xix éste quedó reducido a una pequeña cuña entre Guadalajara y Cuenca, y en 1822 se desplazó al sector de Tarancón y Barajas de Melo. La inclusión de estos últimos pueblos en Cuenca no se verificó hasta la versión definitiva, elaborada tras la intervención de los altos tribunales y decretada en 1833. Hay que observar que alguna de estas localidades había solicitado su agregación a Madrid en el Trienio ${ }^{47}$.

47 Barajas de Melo, Leganiel e Illana en DSC: 30-XII-1821 y 3-I- 1822; Estremera en AC: leg. 78, n. 74, lista de peticiones de 7-IV-1821. Ortego (1991, vol. II, p. 904) señala que, tras la división provincial de 1833, Illana quiso pasar a Cuenca, de la que ya había formado parte desde principios de siglo. 
Finalmente debemos apuntar dos rectificaciones en la delimitación madrileña con posterioridad al decreto de Javier de Burgos. Aranjuez, inicialmente agregada al partido toledano de Ocaña por estar al sur del Tajo, se reintegró a Madrid en fecha no determinada, pero anterior en todo caso a 1842. Por último, en 1850 se incorporó también la pequeña localidad guadalajareño de Valdeavero, que ya lo había solicitado en $1821^{48}$.

\section{BIBLIOGRAFÍA}

Berazaluce, Ana M. (1966-67): Arias Teijeiro. Diarios (1828-1831), Universidad de Navarra, Pamplona.

BuRgueño, Jesús (1994): La formación de la división territorial de España (1800-1850), tesis doctoral microfichada n. 2209, Universidad de Barcelona.

- (1996): Geografía política de la España constitucional. La división provincial, Centro de Estudios Constitucionales, Madrid.

CABallero, Fermín (1834): «División judicial», Eco del Comercio, n. 16 de mayo, Madrid

CARPio Martín, José (1977): «La provincia de Albacete entre las antiguas organizaciones territoriales y los futuros proyectos regionales», Al-basit, n. 4, pp. 42-54, Albacete.

Chavarri Sidera, Pilar (1988): Las elecciones de diputados a las Cortes generales y extraordinarias (1810-1813), Centro de Estudios Constitucionales, Madrid.

FontAna LÁZARo, Josep (1983): La crisis del antiguo régimen, 1808-1833, Crítica, Barcelona.

García Martín, Francisco (1988): «Análisis, comentario y documentación de las divisiones administrativas que ha sufrido la región. Siglo XIX», Primer congreso de historia de Castilla-La Mancha, vol. X-2, pp. 397-426, Junta de Comunidades, Toledo.

GuAITA, Aurelio (1975): División territorial y descentralización, Inst. Estudios Administración Local, Madrid.

Jiménez MontESERÍ, Miguel (1976): «Los orígenes de la provincia de Cuenca como entidad administrativa», Cuenca, n. $10,12 \mathrm{pp} . \mathrm{s} / \mathrm{n}$.

LARRAmendi, José Agustín de (1858-59): «Informe sobre la necesidad de desaguar las lagunas de La Mancha en beneficio de la pública salud y de la agricultura», Revista de obras públicas. Colección de memorias y documentos, vol. III, pp. 45-82, original de 1807, Madrid.

LÓPEZ GÓmez, Antonio (1978): «Las obras geográficas de Fermín Caballero», Arbor, vol. XCIX, n. 386, pp. 37-59, Madrid.

Melón, Amando (1961): «Notas sobre el municipio y antigua provincia de Madrid, Esta dios geográficos, n. 84-85, pp. 325-352, Madrid.

Miñano, Sebastián de (1826-29): Diccionario geográfico-estadístico de España y Portugal, Imp. Pierart-Peralta, 11 vols., Madrid.

Marcos Gonzalez, M. Dolores (1971): Castilla la Nueva y Extremadura, M. Artola (dir.), La España del antiguo régimen, n. 6, Universidad de Salamanca.

Merino Álvarez, Abelardo (1978): Geografía histórica del territorio de la actual provincia de Murcia, Academia de Alfonso X el Sabio, Murcia.

48 AC: leg. 78, n. 74, 29-IV-1821. Es Aurelio Guaita (1975, p. 83) quien da noticia de esta modificación. 
Muñoz de SAn Pedro, Miguel (1960-64): «Extremadura en 1829. Noticias de sus partidos y localidades», Revista de Estudios Extremeños, vol. XVI (pp. 31-58), XIX (5-49) у XX (5-52).

ÑACle Garcí, Ángel (1990): La antigua provincia de Chinchilla y la creación de la provincia de Albacete, Ayto. de Chinchilla, Chinchilla.

Ortego Gil, Pedro (1990): Evolución legislativa de 1a Diputación provincial en España, 1812-1845. La Diputación provincial de Guadalajara, Universidad Complutense, Madrid.

Pillet Capdepón, Félix (1984): Geografía urbana de Ciudad Real (1255-1980), Akal, Torrejón de Ardoz.

Resumen: La reforma de la división provincial en la Meseta Meridional (1800-1850). La división provincial española actual tiene su origen en el cambio de régimen político que se produjo a lo largo de la primera mitad del siglo xIx. Como resultado de este complejo proceso, inspirado en principios racionalistas y homogeneizadores, los antiguos límites de las provincias de Castilla la Nueva sufrieron cambios muy importantes, al tiempo que se formó una nueva provincia a caballo de tierras anteriormente castellanas y murcianas, con capital en Albacete.

Palabras Clave: Provincia. Castilla-La Mancha. Madrid. Siglo XIX.

ABSTRACT: The reform of the provincial division in the spanish southern plateau (18001850). The present provincial division of Spain was born in the political change that happened all along the first half of the 19th century. As result of this complex process, inspirad on racionalist ideas and with finalities of homogeneity, the old boundaries of New Castile provinces were thoroughly modified, and at the same time a new province was mude with lands from Castile and Murcia, with Albacete as a chief town.

Key words: Province. Castile-La Mancha. Madrid, 19th century.

Résumé: La réforme de la division provinciale au plateau méridional espagnole (18001850). L'actuelle division provinciale (ou départementale) espagnole a son origine au change de régime politique réalisé pendant la première moitié du $\mathrm{XIX}^{\mathrm{e}}$ siècle. Des résultats de cette complexe opération, inspirée en idées de racionalité et homogénéité, furent l'important bouleversement des anciennes limites provinciales de la Nouvelle Castille et aussi la iormation d'une nouvelle province avec territoires de Castille et mème de Murcie, dont chief-lieu c'est Albacete.

Mots CLÉ: Province. Castille-La Manche. Madrid. XIX ${ }^{\mathrm{e}}$ siècle. 